\title{
SOME PROPERTIES OF SOLUTIONS OF ITÔ EQUATIONS WITH DRIFT IN $L_{d+1}$
}

\author{
N.V. KRYLOV
}

\begin{abstract}
This paper is a natural continuation of [8], where strong Markov processes are constructed in time inhomogeneous setting with Borel measurable uniformly bounded and uniformly nondegenerate diffusion and drift in $L_{d+1}\left(\mathbb{R}^{d+1}\right)$. Here we study some properties of these processes such as higher summability of Green's functions, boundedness of resolvent operators in Lebesgue spaces, establish Itô's formula, and so on.
\end{abstract}

\section{INTRODUCTION}

Let $\mathbb{R}^{d}$ be a Euclidean space of points $x=\left(x^{1}, \ldots, x^{d}\right), d \geq 2$. Fix some $p_{0}, q_{0} \in[1, \infty)$ such that

$$
\frac{d}{p_{0}}+\frac{1}{q_{0}}=1
$$

It is proved in [8] that Itô's stochastic equations of the form

$$
x_{t}=x+\int_{0}^{t} \sigma\left(t+s, x_{s}\right) d w_{s}+\int_{0}^{t} b\left(t+s, x_{s}\right) d s
$$

admit weak solutions, where $w_{s}$ is a $d$-dimensional Wiener process, $\sigma$ is a uniformly nondegenerate, bounded, Borel function with values in the set of symmetric $d \times d$ matrices, $b$ is a Borel measurable $\mathbb{R}^{d}$ - valued function given on $\mathbb{R}^{d+1}=(-\infty, \infty) \times \mathbb{R}^{d}$ such that

$$
\int_{\mathbb{R}}\left(\int_{\mathbb{R}^{d}}|b(t, x)|^{p_{0}} d x\right)^{q_{0} / p_{0}} d t<\infty
$$

if $p_{0} \geq q_{0}$ or

$$
\int_{\mathbb{R}^{d}}\left(\int_{\mathbb{R}}|b(t, x)|^{q_{0}} d t\right)^{p_{0} / q_{0}} d x<\infty
$$

if $p_{0} \leq q_{0}$. Observe that the case $p_{0}=q_{0}=d+1$ is not excluded and in this case the condition becomes $b \in L_{d+1}\left(\mathbb{R}^{d+1}\right)$.

The goal of this article is to study the properties of such solutions or Markov processes whose trajectories are solutions of (1.2). In particular, in Section 2 for more or less general processes of diffusion type we derive

2010 Mathematics Subject Classification. 60H10, 60J60.

Key words and phrases. Itô's equations with singular drift, Markov diffusion processes, transport equation. 
several estimates of Aleksandrov type by using Lebesgue spaces with mixed norms like (in case $t=0, x=0$ in (1.2))

$$
E \int_{0}^{\infty} e^{-t} f\left(t, x_{t}\right) d t \leq N\|f\|_{L_{p, q}},
$$

provided that $d / p+1 / q \leq 1$.

We also show that expected time when the process $\left(t, x_{t}\right)$, starting at $(0,0)$, exits from $\left[0, R^{2}\right) \times\{x:|x|<R\}$ is comparable to $R^{2}$. This plays a crucial role in Section 3 where we show a significant improvement of the Aleksandrov estimates in the direction of lowering the powers of summability of $f$ in (1.5) to $d_{0} / p+1 / q \leq 1$ with $d_{0}<d$. Time homogeneous versions of these estimates are also given.

In the same Section 2 we give some estimates of the distribution of the exit times from cylinders, which are heavily used in the sequel. In particular, we prove that, for any $0 \leq s \leq t<\infty$,

$$
E \sup _{r \in[s, t]}\left|x_{r}-x_{s}\right|^{n} \leq N|t-s|^{n / 2},
$$

regardless of wether $|t-s|$ is small or large. It is to be said that instead of (1.3) or (1.4), which are not invariant under self-similar transformations, we impose a slightly stronger assumption on $b$, that is invariant.

As we mentioned above, in Section 3 we improve the results of Section 2 in what concerns the Aleksandrov estimates, which allows us to prove Itô's formula for $W_{p, q}^{1,2}(Q)$-functions if $d_{0} / p+1 / q \leq 1$.

Section 4 is devoted to the case when our process is, actually, not just of diffusion type, but a Markov (time-inhomogeneous) process, whose existence is shown in [8]. We prove that their resolvent operators are bounded in $L_{p, q}$.

In the final Section 5 we prove the maximum principle for the transport equation $\partial_{t} u+b^{i} D_{i} u=0$ with $b \in L_{p, q}$ and $u$ from a Sobolev class. Results of that kind seem to be new even if $u$ is smooth.

It is worth mentioning that there is a vast literature about stochastic and PDE equations when (1.1) is replaced with $d / p+2 / q \leq 1$. This condition is much stronger than ours. Still we refer the reader to the recent articles [13], [1], [16] and the references therein for the discussion of many powerful and exciting results obtained under this stronger condition. There are also many papers when this condition is considerably relaxed on the account of imposing various regularity conditions on $\sigma$ and $b$ and/or considering random initial conditions with bounded density, see, for instance, [18], [17] and the references therein. Restricting the situation to the one when $\sigma$ and $b$ are independent of time allows one to relax the above conditions significantly further, see, for instance, [7] and the references therein.

Introduce

$$
\begin{gathered}
B_{R}=\left\{x \in \mathbb{R}^{d}:|x|<R\right\}, \quad B_{R}(x)=x+B_{R}, \quad C_{T, R}=[0, T) \times B_{R}, \\
C_{T, R}(t, x)=(t, x)+C_{T, R}, \quad C_{R}(t, x)=C_{R^{2}, R}(t, x), \quad C_{R}=C_{R}(0,0),
\end{gathered}
$$




$$
D_{i}=\frac{\partial}{\partial x^{i}}, \quad D_{i j}=D_{i} D_{j} \quad \partial_{t}=\frac{\partial}{\partial t} .
$$

For $p, q \in[1, \infty]$ and domains $Q \subset \mathbb{R}^{d+1}$ we introduce the space $L_{p, q}(Q)$ as the space of Borel functions on $Q$ such that

$$
\|f\|_{L_{p, q}(Q)}^{q}:=\int_{\mathbb{R}}\left(\int_{\mathbb{R}^{d}} I_{Q}(t, x)|f(t, x)|^{p} d x\right)^{q / p} d t<\infty
$$

if $p \geq q$ or

$$
\|f\|_{L_{p, q}(Q)}^{p}:=\int_{\mathbb{R}^{d}}\left(\int_{\mathbb{R}} I_{Q}(t, x)|f(t, x)|^{q} d t\right)^{p / q} d x<\infty
$$

if $p \leq q$ with natural interpretation of these definitions if $p=\infty$ or $q=\infty$. If $Q=\mathbb{R}^{d+1}$, we drop $Q$ in the above notation. Observe that $p$ is associated with $x$ and $q$ with $t$ and the interior integral is always elevated to the power $\leq 1$. In case $p=q=d+1$ we abbreviate $L_{d+1, d+1}=L_{d+1}$. For the set of functions on $\mathbb{R}^{d}$ summable to the $p$ th power we use the notation $L_{p}\left(\mathbb{R}^{d}\right)$.

If $\Gamma$ is a measurable subset of $\mathbb{R}^{d+1}$ we denote by $|\Gamma|$ its Lebesgue measure. The same notation is used for measurable subsets of $\mathbb{R}^{d}$ with $d$-dimensional Lebesgue measure. We hope that it will be clear from the context which Lebesgue measure is used. If $\Gamma$ is a measurable subset of $\mathbb{R}^{d+1}$ and $f$ is a function on $\Gamma$ we denote

$$
f_{\Gamma} f d x d t=\frac{1}{|\Gamma|} \int_{\Gamma} f d x d t
$$

In case $f$ is a function on a measurable subset $\Gamma$ of $\mathbb{R}^{d}$ we set

$$
f_{\Gamma} f d x=\frac{1}{|\Gamma|} \int_{\Gamma} f d x
$$

\section{The CASE OF General DIFfUSion type PROCESSES WITH DRIFT IN}

$$
L_{p_{0}, q_{0}}
$$

Let $(\Omega, \mathcal{F}, P)$ be a complete probability space, let $\mathcal{F}_{t}, t \geq 0$, be an increasing family of complete $\sigma$-fields $\mathcal{F}_{t} \subset \mathcal{F}$, let $m_{t}$ be an $\mathbb{R}^{d}$-valued continuous local martingale relative to $\mathcal{F}_{t}$, let $A_{t}$ be a continuous $\mathcal{F}_{t}$-adapted nondecreasing process, let $B_{t}$ be a continuous $\mathbb{R}^{d}$-valued $\mathcal{F}_{t}$-adapted process which has finite variation (a.s.) on each finite time interval and such that $d\left|B_{t}\right| \ll d A_{t}$. Assume that

$$
A_{0}=0, \quad m_{0}=B_{0}=0, \quad d\langle m\rangle_{t} \ll d A_{t}
$$

and that we are also given progressively measurable relative to $\mathcal{F}_{t}$ nonnegative processes $r_{t}$ and $c_{t}$. Finally, introduce

$$
\begin{gathered}
x_{t}=m_{t}+B_{t}, \quad \mathrm{t}_{t}=\int_{0}^{t} r_{s} d A_{s}, \quad \phi_{t}=\int_{0}^{t} c_{s} d A_{s}, \\
a_{t}^{i j}=\frac{1}{2} \frac{d\left\langle m^{i}, m^{j}\right\rangle_{t}}{d A_{t}}, \quad b_{t}=\frac{d B_{t}}{d A_{t}} .
\end{gathered}
$$


Also take a stopping time $\gamma$ and denote

$$
A=E \int_{0}^{\gamma} e^{-\phi_{t}} \operatorname{tr} a_{s} d A_{t}, \quad B=E \int_{0}^{\gamma} e^{-\phi_{t}}\left|d B_{t}\right| .
$$

Assumption 2.1. We are given a function $h \in L_{p_{0}, q_{0}, \text { loc }}$ such that

$$
\left|b_{t}\right| \leq r_{t}^{1 / q_{0}}\left(\operatorname{det} a_{t}\right)^{1 / p_{0}} h\left(\mathrm{t}_{t}, x_{t}\right)
$$

Furthermore, there exists a constant $\bar{H}>0$ and a continuous function $W(\theta) \geq 0, \theta \in[0,1]$, such that $W(0)=0, W(1)=1$ and for any $(t, x) \in$ $\mathbb{R}^{d+1}, \rho>0$, and $\tau \in\left(0, \rho^{2}\right]$ we have

$$
\|h\|_{L_{p_{0}, q_{0}}\left(C_{\tau, \rho}(t, x)\right)}^{q_{0}} \leq \bar{H} \rho W\left(\tau / \rho^{2}\right) .
$$

Remark 2.1. The reader will see that the assumption that $W(0)=0$ can be replaced by the requirement that $W(0)$ be sufficiently small, the smallness defined only by $d, p_{0}, \bar{H}, W$, and $\delta$, where $\delta$ is introduced in Assumption 2.2 below.

Observe that if $p_{0}=q_{0}=d+1$ and $h \in L_{d+2}$ (a typical case in the theory of parabolic equations), then (2.1) is satisfied with $\bar{H}=\|h\|_{L_{d+2}}^{d+1}$, because by Hölder's inequality

$$
\|h\|_{L_{d+1}\left(C_{\tau, \rho}(t, x)\right)}^{d+1} \leq \bar{H} \rho(\tau / \rho)^{2 /(d+2)} .
$$

On the other hand, it may happen that (2.1) is satisfied with $p_{0}=q_{0}=d+1$ but $h \notin L_{d+2, \text { loc }}$.

It is worth noting that a condition very similar to (2.1) first appeared in $[14]$.

Example 2.1. Take $\alpha \in(0, d), \beta \in(0,1)$ such that $\alpha+2 \beta=d+1$ and consider the function $g(t, x)=|t|^{-\beta}|x|^{-\alpha}$. Observe that

$$
\int_{C_{\tau, \rho}(t, x)} g(s, y) d y d s=\rho\left(\tau / \rho^{2}\right)^{1-\beta} \int_{C_{1}\left(t^{\prime}, x^{\prime}\right)} g(s, y) d y d s,
$$

where $t^{\prime}=t / \tau, x^{\prime}=x / \rho$. Obviously, the last integral is a bounded function of $\left(t^{\prime}, x^{\prime}\right)$. Hence, the function $h=g^{1 /(d+1)}$ satisfies $(2.1)$ with $p_{0}=q_{0}=$ $d+1$. As is easy to see for any $p>d+1$ one can find $\alpha$ and $\beta$ above such that $h \notin L_{p, \text { loc }}$.

The following is a particular case of Theorem 4.5 of [8]. In Theorem 2.1 $p_{0}=\infty$ is allowed (and then $q_{0}=1$ ).

Theorem 2.1. Suppose that Assumption 2.1 is satisfied and

$$
p, q \in[1, \infty], \quad \frac{d}{p}+\frac{1}{q}=1 .
$$

Then for any Borel $f \geq 0$

$$
E \int_{0}^{\gamma} e^{-\phi_{t}} \kappa_{t} f\left(\mathrm{t}_{t}, x_{t}\right) d A_{t} \leq N\left(d, p_{0}\right)\left(A+\|h\|_{L_{p_{0}, q_{0}}}^{2 q_{0}}\right)^{d /(2 p)}\|f\|_{L_{p, q}},
$$

where $\kappa_{t}=r_{t}^{1 / q}\left(\operatorname{det} a_{t}\right)^{1 / p}$. 
Our first goal is to estimate $A$ and eliminate it from (2.3).

Assumption 2.2. We have $t_{\infty}=\infty$ and there is a constant $\delta \in(0,1)$ such that for all values of the arguments

$$
\delta \operatorname{tr} a_{t} \leq r_{t} .
$$

This assumption and Assumption 2.1 are supposed to hold below in this section.

\section{Lemma 2.2. Introduce}

$$
\tau_{R}=\inf \left\{t \geq 0:\left(\mathrm{t}_{t}, x_{t}\right) \notin C_{R}\right\} .
$$

Then

$$
A:=E \int_{0}^{\tau_{R}} \operatorname{tr} a_{t} d A_{t} \leq \delta^{-1} R^{2}
$$

and consequently (for $c_{t} \equiv 0$ ), assuming (2.2), for any Borel nonnegative $f$

$$
E \int_{0}^{\tau_{R}} \kappa_{t} f\left(\mathrm{t}_{t}, x_{t}\right) d A_{t} \leq N\left(d, p_{0}, \delta\right)\left(1+\bar{H}^{d / p}\right) R^{d / p}\|f\|_{L_{p, q}} .
$$

In particular (as $p=q=d+1$ ),

$$
E \int_{0}^{\tau_{R}}\left(r_{t} \operatorname{det} a_{t}\right)^{1 /(d+1)} d A_{t} \leq N\left(d, p_{0}, \bar{H}, \delta\right) R^{2} .
$$

Proof. We have

$$
\int_{0}^{\tau_{R}} \operatorname{tr} a_{t} d A_{t} \leq \delta^{-1} \int_{0}^{\tau_{R}} r_{t} d A_{t}=\delta^{-1} \mathrm{t}_{\tau_{R}} \leq \delta^{-1} R^{2}
$$

and (2.4) follows. After that (2.5) follows from (2.1) and (2.3) with $c=0$, $f=I_{C_{R}}$, and $h$ replaced by $h I_{C_{R}}$. The lemma is proved.

Remark 2.2. The detailed form of the right-hand side in (2.5) will be important in Section 5 .

Estimate (2.6) says that in the typical case of nondegenerate diffusion $\tau_{R}$ is of order not more than $R^{2}$. A very important fact which is implied by Corollary 2.6 below is that $\tau_{R}$ is of order not less than $R^{2}$. On the way of showing this we start with the following.

Theorem 2.3. There is $\theta=\theta\left(d, \delta, p_{0}, \bar{H}, W\right)>0$ such that for all $R>0$

$$
P\left(\mathrm{t}_{\tau_{R}}>\theta R^{2}\right) \geq 1 / 2 \text {. }
$$

Proof. First we make an important claim, having a general character, that it suffices to prove (2.7) for $R=1$. To prove the claim, fix an $R>0$ and make a self-similar transformation by setting $\hat{x}_{t}=x_{t} / R, \hat{\mathrm{t}}_{t}=\mathrm{t}_{t} / R^{2}$. Then

where

$$
\hat{x}_{t}=\hat{m}_{t}+\hat{B}_{t}, \quad \hat{\mathrm{t}}_{t}=\int_{0}^{t} \hat{r}_{s} d A_{s},
$$

$$
\hat{m}_{t}=m_{t} / R, \quad \hat{B}_{t}=B_{t} / R, \quad \hat{r}_{t}=r_{t} / R^{2} .
$$


Observe that

$$
\hat{a}_{t}^{i j}=\frac{1}{2} \frac{d\left\langle\hat{m}^{i}, \hat{m}^{j}\right\rangle_{t}}{d A_{t}}=a_{t}^{i j} / R^{2}, \quad \delta \operatorname{tr} \hat{a}_{t} \leq \hat{r}_{t}
$$

and for $\hat{b}_{t}=d \hat{B}_{t} / d A_{t}$ and $\hat{h}(t, x)=R h\left(R^{2} t, R x\right)$

$$
\left|\hat{b}_{t}\right|=\left|b_{t}\right| / R \leq \hat{r}_{t}^{1 / q_{0}}\left(\operatorname{det} \hat{a}_{t}\right)^{1 / p_{0}} \hat{h}\left(\hat{\mathrm{t}}_{t}, \hat{x}_{t}\right) .
$$

Furthermore, if $p_{0} \geq q_{0}$, for any $\rho>0, \tau \leq \rho^{2}$, and $\left(t_{0}, x_{0}\right) \in \mathbb{R}^{d+1}$

$$
\begin{aligned}
& \int_{0}^{\tau}\left(\int_{|x| \leq \rho}\left|\hat{h}\left(t_{0}+t, x_{0}+x\right)\right|^{p_{0}} d x\right)^{q_{0} / p_{0}} d t \\
& =R^{q_{0}-2-q_{0} d / p_{0}}\|h\|_{L_{p_{0}, q_{0}}\left(C_{R^{2} \tau, R \rho}\left(R^{2} t_{0}, R x_{0}\right)\right)}^{q_{0}} \\
& \leq R^{q_{0}-2-q_{0} d / p_{0}} \bar{H} R \rho W\left(\tau / \rho^{2}\right)=\bar{H} \rho W\left(\tau / \rho^{2}\right) .
\end{aligned}
$$

Also, if $q_{0}>p_{0}$,

$$
\begin{gathered}
\int_{|x| \leq \rho}\left(\int_{0}^{\tau}\left|\hat{h}\left(t_{0}+t, x_{0}+x\right)\right|^{q_{0}} d t\right)^{p_{0} / q_{0}} d x \\
=R^{p_{0}-2 p_{0} / q_{0}-d}\|h\|_{L_{p_{0}, q_{0}}\left(C_{R^{2} \tau, R \rho}\left(R^{2} t_{0}, R x_{0}\right)\right)}^{p_{0}} \\
\leq R^{p_{0}-2 p_{0} / q_{0}-d}\left(\bar{H} R \rho W\left(\tau / \rho^{2}\right)\right)^{p_{0} / q_{0}}=\left(\bar{H} \rho W\left(\tau / \rho^{2}\right)\right)^{p_{0} / q_{0}} .
\end{gathered}
$$

Hence, if (2.7) for $R=1$ is true, then

$$
P\left(\hat{\mathrm{t}}_{\hat{\tau}_{1}}>\theta\right) \geq 1 / 2 \text {, }
$$

where

$$
\hat{\tau}_{1}=\inf \left\{t \geq 0:\left(\hat{\mathrm{t}}_{t}, \hat{x}_{t}\right) \notin C_{1}\right\}=\inf \left\{t \geq 0:\left(\mathrm{t}_{t}, x_{t}\right) \notin C_{R}\right\}=\tau_{R},
$$

so that (2.8) is just another form of (2.7).

Having proved the claim we set $R=1$ and observe that by (2.5)

$$
\begin{gathered}
E \int_{0}^{\tau_{1}}\left|b\left(\mathrm{t}_{t}, x_{t}\right)\right| I_{\mathrm{t}_{t} \leq \theta} d A_{t} \leq N\left(d, p_{0}, \delta\right)\left(1+\bar{H}^{d / p_{0}}\right)\|h\|_{L_{p_{0}, q_{0}}\left(C_{\theta, 1}\right)} \\
\leq N\left(d, p_{0}, \delta\right)\left(1+\bar{H}^{d / p_{0}}\right)[\bar{H} W(\theta)]^{1 / q_{0}} .
\end{gathered}
$$

Furthermore, for $\gamma=\inf \left\{t \geq 0: \mathrm{t}_{t} \geq \theta\right\}$

$$
\begin{gathered}
E \sup _{t \leq \gamma}\left|m_{t}\right| \leq 3 E\langle m\rangle_{\gamma}^{1 / 2} \leq 6 E\left(\int_{0}^{\gamma} \operatorname{tr} a_{t} d A_{t}\right)^{1 / 2} \\
\leq(6 / \delta) E\left(\int_{0}^{\gamma} r_{t} d A_{t}\right)^{1 / 2} \leq(6 / \delta) \sqrt{\theta} .
\end{gathered}
$$

It follows that for $\theta$ small enough

$$
E \sup _{t \leq \tau_{1} \wedge \gamma}\left|x_{t}\right| \leq 1 / 2, \quad P\left(\sup _{t \leq \tau_{1} \wedge \gamma}\left|x_{t}\right| \geq 1\right) \leq 1 / 2, \quad P\left(\sup _{t \leq \tau_{1} \wedge \gamma}\left|x_{t}\right|<1\right) \geq 1 / 2,
$$

and (2.7) follows. The theorem is proved.

Corollary 2.4. For $\lambda \in[0,1]$ and $R>0$ we have

$$
E e^{-\lambda R^{-2} \mathbf{t}_{\tau_{R}}} \leq e^{-\lambda \theta / 4} \text {. }
$$


Indeed, self-similarity allows us to concentrate on $R=1$. In that case the derivative with respect to $\lambda$ of the left-hand side of (2.9) is

$$
-E \mathrm{t}_{\tau_{R}} e^{-\lambda \mathrm{t}_{\tau_{1}}} \leq-e^{-\lambda} E \mathrm{t}_{\tau_{1}} \leq-e^{-\lambda} \theta / 2,
$$

where the last inequality follows from (2.7). By integrating we find

$$
E e^{-\lambda \mathbf{t}_{\tau_{1}}}-1 \leq\left(e^{-\lambda}-1\right) \theta / 2,
$$

which after using

$$
e^{-\lambda}-1 \leq-\lambda / 2, \quad 1-\lambda \theta / 4 \leq e^{-\lambda \theta / 4}
$$

leads to $(2.9)$.

Theorem 2.5. For any $\lambda, R>0$ we have

$$
E e^{-\lambda \mathbf{t}_{\tau_{R}}} \leq e^{\theta / 4} e^{-\sqrt{\lambda} R \theta / 4}
$$

In particular, for any $R, t \in(0, \infty)$ we have

$$
P\left(\mathrm{t}_{\tau_{R}} \leq t\right) \leq e^{\theta / 4} \exp \left(-\frac{\theta^{2} R^{2}}{64 t}\right) .
$$

Proof. We may assume that $R=1$. In that case take an integer $n \geq 1$, introduce $\tau^{k}, k=1, \ldots, n$, as the first exit time of $\left(\mathrm{t}_{t}, x_{t}\right)$ from $C_{1 / n}\left(\mathrm{t}_{\tau^{k-1}}, x_{\tau^{k-1}}\right)$ after $\tau^{k-1}\left(\tau^{0}:=0\right)$. In light of $(2.9)$ we have

$$
E\left(e^{-n^{2}\left(\mathrm{t}_{\tau^{k}}-\mathrm{t}_{\tau^{k-1}}\right)} \mid \mathcal{F}_{\tau^{k-1}}\right) \leq e^{-\theta / 4} .
$$

Hence

$$
E e^{-n^{2} \mathbf{t}_{\tau_{1}}} \leq E \prod_{k=1}^{n} e^{-n^{2}\left(\mathrm{t}_{\tau^{k}}-\mathrm{t}_{\tau^{k-1}}\right)} \leq e^{-n \theta / 4},
$$

which for $n=\lfloor\sqrt{\lambda}\rfloor$ yields $(2.10)$.

We prove (2.11) again assuming that $R=1$. We have

$$
P\left(\mathrm{t}_{\tau_{1}} \leq t\right)=P\left(\exp \left(-\lambda \mathrm{t}_{\tau_{1}} \geq \exp (-\lambda t)\right) \leq e^{\theta / 4} \exp (\lambda t-\sqrt{\lambda} \theta / 4) .\right.
$$

For $\sqrt{\lambda}=\theta /(8 t)$ we get $(2.11)$. The theorem is proved.

Corollary 2.6. There is a constant $N=N(\theta)$ such that for any $R \in(0, \infty)$

$$
N E \mathrm{t}_{\tau_{R}} \geq R^{2}, \quad N E \int_{0}^{\tau_{R}} e^{-\mathrm{t}_{t}} r_{t} d A_{t} \geq R^{2} \wedge 1 .
$$

Indeed, for any $t_{0}>0$

$$
\begin{gathered}
E \mathrm{t}_{\tau_{R}} \geq t_{0} R^{2} P\left(\mathrm{t}_{\tau_{R}}>t_{0} R^{2}\right) \geq R^{2} t_{0}\left(1-e^{\theta / 4} \exp \left(-\frac{\theta^{2}}{64 t_{0}}\right)\right), \\
E \int_{0}^{\tau_{R}} e^{-\mathrm{t}_{t}} r_{t} d A_{t}=E\left(1-e^{-\mathrm{t}_{\tau_{R}}}\right) \geq E I_{\mathrm{t}_{\tau_{R}}>t_{0} R^{2}}\left(1-e^{-t_{0} R^{2}}\right) \\
=P\left(\mathrm{t}_{\tau_{R}}>t_{0} R^{2}\right)\left(1-e^{-t_{0} R^{2}}\right) \\
\geq\left(1-e^{\theta / 4} \exp \left(-\frac{\theta^{2}}{64 t_{0}}\right)\right)\left(1-e^{-t_{0} R^{2}}\right),
\end{gathered}
$$


which yields (2.12) for an appropriate $t_{0}$.

Corollary 2.7. Assume that $\mathrm{t}_{t} \leq \delta^{-1} t$ for all $t \geq 0$. Then for any $n>0$ and $0 \leq s \leq t$ we have

$$
E \sup _{r \in[s, t]}\left|x_{r}-x_{s}\right|^{n} \leq N|t-s|^{n / 2},
$$

where $N=N(n, \delta, \theta)$.

Indeed, clearly we may assume that $s=0$. Then

$$
\begin{aligned}
E \sup _{r \leq t]}\left|x_{r}\right|^{n}= & n \int_{0}^{\infty} \mu^{n-1} P\left(\sup _{r \leq t}\left|x_{r}\right| \geq \mu\right) d \mu \leq n \int_{0}^{t^{1 / 2}} \mu^{n-1} d \mu \\
& +e^{\theta / 4} \int_{t^{1 / 2}}^{\infty} \mu^{n-1} \exp \left(-\frac{\delta \theta^{2} \mu^{2}}{64 t}\right) d \mu,
\end{aligned}
$$

where the inequality is due to (2.11) and the fact that, for $\mu>t^{1 / 2}$,

$$
\left\{\sup _{r \leq t}\left|x_{r}\right| \geq \mu\right\} \subset\left\{\tau_{\mu} \leq t\right\} \subset\left\{\mathrm{t}_{\tau_{\mu}} \leq t / \delta\right\} .
$$

Changing variables $\mu=\nu \sqrt{t}$ leads to (2.13).

Remark 2.3. Observe that the right-hand side of (2.13) has the same form for small $|t-s|$ and for large ones.

Now we start moving toward estimating the resolvent of Markov diffusion processes in $L_{p, q}$.

Lemma 2.8. Assume (2.2). Then there is a constant $N$, depending only on $\delta, d, \bar{H}, p, p_{0}, W$, such that for any $t_{0} \geq 0, x_{0} \in \mathbb{R}^{d}$, and Borel nonnegative $f$ vanishing outside $C_{1}\left(t_{0}, x_{0}\right)$ we have

$$
E \int_{0}^{\infty} e^{-\mathrm{t}_{t}} f\left(\mathrm{t}_{t}, x_{t}\right) \kappa_{t} d A_{t} \leq N \Phi\left(t_{0}, x_{0}\right)\|f\|_{L_{p, q}}
$$

where $\kappa_{t}=r_{t}^{1 / q}\left(\operatorname{det} a_{t}\right)^{1 / p}$ and $\Phi(t, x)=\exp (-(|x|+\sqrt{t}) \theta / 8)$.

Proof. Fix $\rho=\rho(\theta)>0$ such that the right-hand side of (2.11) equals $1 / 2$ when $R=\rho$ and $t=1$. Then introduce $\tau^{0}$ as the first time $\left(\mathrm{t}_{t}, x_{t}\right)$ hits $\bar{C}_{1}\left(t_{0}, x_{0}\right)$ and set $\gamma^{0}$ as the first time after $\tau^{0}$ the process $\left(\mathrm{t}_{t}, x_{t}\right)$ exits from $C_{1,1+\rho}\left(t_{0}, x_{0}\right)$. We define recursively $\tau^{k}, k=1,2, \ldots$, as the first time after $\gamma^{k-1}$ the process $\left(\mathrm{t}_{t}, x_{t}\right)$ hits $\bar{C}_{1}\left(t_{0}, x_{0}\right)$ and $\gamma^{k}$ as the first time after $\tau^{k}$ the process $\left(\mathrm{t}_{t}, x_{t}\right)$ exits from $C_{1,1+\rho}\left(t_{0}, x_{0}\right)$.

These stopping times are either infinite or lie between $t_{0}$ and $t_{0}+1$. Therefore the left-hand side of (2.14) equals

$$
E \sum_{k=0}^{\infty} e^{-\mathrm{t}_{\tau^{k}}} I_{k},
$$


where

$$
I_{k}=I_{\tau^{k} \in\left[t_{0}, t_{0}+1\right]} E\left(\int_{\tau^{k} \wedge\left(t_{0}+1\right)}^{\gamma^{k} \wedge\left(t_{0}+1\right)} e^{-\left(\mathrm{t}_{t}-\mathrm{t}_{\tau^{k}}\right)} f\left(\mathrm{t}_{t}, x_{t}\right) \kappa_{t} d A_{t} \mid \mathcal{F}_{\tau^{k}}\right) .
$$

Here

$$
\begin{gathered}
\int_{\tau^{k} \wedge\left(t_{0}+1\right)}^{\gamma^{k} \wedge\left(t_{0}+1\right)} e^{-\left(\mathrm{t}_{t}-\mathrm{t}_{\tau^{k}}\right)} \operatorname{tr} a_{t} d A_{t} \leq \delta^{-1} \int_{\tau^{k} \wedge\left(t_{0}+1\right)}^{\gamma^{k} \wedge\left(t_{0}+1\right)} r_{t} d A_{t} \\
=\delta^{-1}\left(\mathrm{t}_{\gamma^{k} \wedge\left(t_{0}+1\right)}-\mathrm{t}_{\tau^{k} \wedge\left(t_{0}+1\right)}\right) \leq \delta^{-1} .
\end{gathered}
$$

It follows from (2.3) that $I_{k} \leq N\|f\|_{L_{p, q}}$.

Next, observe that, if $\sqrt{t_{0}}>\left|x_{0}\right|$, then $\tau^{0}$ is bigger than the first exit time of $\left(\mathrm{t}_{t}, x_{t}\right)$ from $C_{\sqrt{t_{0}}}$, and by Theorem 2.5

$$
E e^{-\mathrm{t}_{\tau} 0} \leq N e^{-\sqrt{t_{0}} \theta / 4} .
$$

In case $\sqrt{t_{0}} \leq\left|x_{0}\right|$ and $\left|x_{0}\right|>1$ our $\tau^{0}$ is bigger than the first exit time of $\left(\mathrm{t}_{t}, x_{t}\right)$ from $C_{\left|x_{0}\right|-1}$, and

$$
E e^{-\mathrm{t}_{\tau^{0}}} \leq N e^{-\left(\left|x_{0}\right|-1\right) \theta / 4} \leq N e^{-\left|x_{0}\right| \theta / 4} .
$$

The last estimate (with different $N$ ) also holds if $\left|x_{0}\right| \leq 1$ and we conclude that in all cases

$$
E e^{-\mathbf{t}_{\tau} 0} \leq N e^{-\left(\sqrt{t_{0}}+\left|x_{0}\right|\right) \theta / 8} .
$$

Furthermore, by the choice of $\rho$

$$
\begin{aligned}
& E\left(e^{-\left(\mathrm{t}_{\gamma^{k}}-\mathrm{t}_{\tau^{k}}\right)} \mid \mathcal{F}_{\tau^{k}}\right) \leq \frac{1}{2}, \\
& E e^{-\mathrm{t}_{\tau^{k}}}=E e^{-\mathrm{t}_{\gamma^{k-1}}} E\left(e^{-\left(\mathrm{t}_{\tau^{k}}-\mathrm{t}_{\gamma^{k-1}}\right)} \mid \mathcal{F}_{\gamma^{k-1}}\right) \leq \frac{1}{2} E e^{-\mathrm{t}_{\gamma^{k-1}}},
\end{aligned}
$$

so that

$$
E e^{-\mathrm{t}_{\tau^{k}}} \leq \frac{1}{4} E e^{-\mathrm{t}_{\tau^{k-1}}}, \quad E e^{-\mathrm{t}_{\tau^{k}}} \leq 4^{-k} E e^{-\mathrm{t}_{\tau^{0}}} .
$$

Recalling (2.15) we see that the left-hand side of (2.14) is dominated by $N \Phi\left(t_{0}, x_{0}\right)\|f\|_{L_{p, q}}$ and the lemma is proved.

The following theorem shows that the time spent by $\left(\mathrm{t}_{t}, x_{t}\right)$ in cylinders $C_{1}(0, x)$ far from the origin decays very fast.

Theorem 2.9. Suppose that

$$
p, q \in[1, \infty], \quad \nu:=1-\frac{d}{p}-\frac{1}{q} \geq 0 .
$$

Then there is a constant $N=N\left(\delta, d, \bar{H}, p, q, p_{0}, W\right)$ such that for any $\lambda>0$ and Borel nonnegative $f$ we have

$$
E \int_{0}^{\infty} e^{-\lambda \mathrm{t}_{t}} f\left(\mathrm{t}_{t}, x_{t}\right) \kappa_{t} r_{t}^{\nu} d A_{t} \leq N \lambda^{-\nu-d /(2 p)}\left\|\Phi_{\lambda}^{1-\nu} f\right\|_{L_{p, q}},
$$

where $\Phi_{\lambda}(t, x)=\exp (-\sqrt{\lambda}(|x|+\sqrt{t}) \theta / 32)$ and $r_{t}^{\nu}:=1$ if $\nu=0$. 
Proof. First assume that $\nu=0$. The self-similarity argument allows us to also assume that $\lambda=1$. In that case take a nonnegative $\zeta \in C_{0}^{\infty}\left(\mathbb{R}^{d+1}\right)$ with support in $C_{1}$ and unit integral and for $(t, x),(s, y) \in \mathbb{R}^{d+1}$ set

$$
f_{s, y}(t, x)=f(t, x) \zeta(t-s, x-y) .
$$

Clearly, due to Lemma 2.8,

$$
\begin{gathered}
I:=E \int_{0}^{\infty} e^{-\mathrm{t}_{t}} f\left(\mathrm{t}_{t}, x_{t}\right) \kappa_{t} d A_{t}=\int_{0}^{\infty} \int_{\mathbb{R}^{d}} E \int_{0}^{\infty} e^{-\mathrm{t}_{t}} f_{s, y}\left(\mathrm{t}_{t}, x_{t}\right) \kappa_{t} d A_{t} d y d s \\
\leq N \int_{0}^{\infty} \int_{\mathbb{R}^{d}} \Phi(s, y)\left\|f_{s, y}\right\|_{L_{p, q}} d y d s .
\end{gathered}
$$

Case $p \geq q$. Then $q<\infty$ and it follows by Hölder's inequality that

$$
\begin{aligned}
I & \leq N\left(\int_{0}^{\infty} \int_{\mathbb{R}^{d}} \Phi^{q / 2}(s, y) \int_{0}^{\infty}\left(\int_{\mathbb{R}^{d}} f_{s, y}^{p}(t, x) d x\right)^{q / p} d t d y d s\right)^{1 / q} \\
= & N\left(\int_{0}^{\infty} d t\left(\int_{0}^{\infty} \int_{\mathbb{R}^{d}} \Phi^{q / 2}(s, y)\left(\int_{\mathbb{R}^{d}} f_{s, y}^{p}(t, x) d x\right)^{q / p} d y d s\right)\right)^{1 / q} \\
& \leq N\left(\int_{0}^{\infty} d t\left(\int_{0}^{\infty} \int_{\mathbb{R}^{d}} \int_{\mathbb{R}^{d}} \Phi^{p / 4}(s, y) f_{s, y}^{p}(t, x) d y d s d x\right)^{q / p}\right)^{1 / q} .
\end{aligned}
$$

We replace $\Phi^{p / 4}(s, y)$ by $\Phi^{p / 4}(t, x)$ taking into account that these values are comparable as long as $\zeta(t-s, x-y) \neq 0$. After that integrating over $d y d s$ leads immediately to (2.17).

Case $p<q$. It follows by Hölder's inequality that

$$
\begin{aligned}
I & \leq N\left(\int_{0}^{\infty} \int_{\mathbb{R}^{d}} \Phi^{p / 2}(s, y) \int_{\mathbb{R}^{d}}\left(\int_{0}^{\infty} f_{s, y}^{q}(t, x) d t\right)^{p / q} d x d y d s\right)^{1 / p} \\
& \leq N\left(\int_{\mathbb{R}^{d}} d x\left(\int_{0}^{\infty} \int_{\mathbb{R}^{d}} \int_{0}^{\infty} \Phi^{q / 4}(s, y) f_{s, y}^{q}(t, x) d t d y d s\right)^{p / q}\right)^{1 / p} .
\end{aligned}
$$

This leads to (2.17) as above. The theorem is proved if $\nu=0$.

If $\nu \in(0,1)$, by Hölder's inequality the left-hand side of $(2.17)$ is dominated by $I_{1} I_{2}$, where

$$
\begin{gathered}
I_{1}^{1 / \nu}=E \int_{0}^{\infty} e^{-\lambda \mathrm{t}_{t}} r_{t} d A_{t}=1 / \lambda \\
I_{2}^{1 /(1-\nu)}=E \int_{0}^{\infty} e^{-\lambda \mathrm{t}_{t}} r_{t}^{1 /(q-\nu q)}\left(\operatorname{det} a_{t}\right)^{1 /(p-\nu p)} f^{1 /(1-\nu)}\left(\mathrm{t}_{t}, x_{t}\right) d A_{t} .
\end{gathered}
$$

Here

$$
\frac{d}{p-p \nu}+\frac{1}{q-q \nu}=1
$$

so that by the case that $\nu=0$

$$
\begin{aligned}
I_{2}^{1 /(1-\nu)} & \leq N \lambda^{-d /(2 p-2 p \nu)}\left\|\Phi_{\lambda} f^{1 /(1-\nu)}\right\|_{L_{p-p \nu, q-q \nu}} \\
& =N \lambda^{-d /(2 p-2 p \nu)}\left\|\Phi_{\lambda}^{1-\nu} f\right\|_{L_{p, q}}^{1 /(1-\nu)} .
\end{aligned}
$$


This leads to (2.17) again. Finally, if $\nu=1$ so that $p=q=\infty$, the left-hand side of (2.17) is obviously dominated by $\lambda^{-1} \sup f$, so that (2.17) holds with $N=1$. The theorem is proved.

By taking $q=\infty$ and $f(t, x)=f(x)$ we come to the following, which extends Corollary 2.4 of [11] to the case of time dependent drift $b \in L_{p_{0}, q_{0}, \text { loc }}$. It is further generalized in a more specific situation by relaxing the restriction on $p$ in Theorem 3.6.

Corollary 2.10. Let $p \in[d, \infty]$. Then for any $\lambda>0$ and Borel nonnegative $f(x)$ we have

$$
E \int_{0}^{\infty} e^{-\lambda \mathrm{t}_{t}} f\left(x_{t}\right)\left(\operatorname{det} a_{t}\right)^{1 / p} r_{t}^{1-d / p} d A_{t} \leq N \lambda^{-1+d /(2 p)}\left\|\Psi_{\lambda}^{d / p} f\right\|_{L_{p}\left(\mathbb{R}^{d}\right)}
$$

where $\Psi_{\lambda}(x)=\exp (-\sqrt{\lambda}|x| \theta / 32)$ and $N=N\left(\delta, d, \bar{H}, p, p_{0}, W\right)$.

Next results are dealing with the exit times of the process $x_{t}$ rather than $\left(\mathrm{t}_{t}, x_{t}\right)$. We will need them while showing an improved integrability of Green's functions.

Corollary 2.11. Suppose that

$$
\delta r_{t}^{d} \leq \operatorname{det} a_{t}
$$

and for $|x|<R$ introduce

$$
\tau_{R}^{\prime}(x)=\inf \left\{t \geq 0: x+x_{t} \notin B_{R}\right\}, \quad \tau_{R}^{\prime}=\tau_{R}^{\prime}(0) .
$$

Then there exists a constant $T=T\left(d, p_{0}, \bar{H}, \delta\right)$ such that

$$
P\left(\mathrm{t}_{\tau_{R}^{\prime}(x)} \geq T R^{2}\right) \leq 1 / 2
$$

To prove this, observe that $\tau_{R}^{\prime}(x) \leq \tau_{2 R}^{\prime}$, which shows that it suffices to prove $(2.20)$ for $x=0$. In that case for $\varepsilon \in(0,1]$ introduce

$$
\tau_{\varepsilon, R}^{\prime}=\inf \left\{t \geq 0:\left(\mathrm{t}_{t}, x_{t}\right) \notin C_{R^{2}, \varepsilon R}\right\} .
$$

Then (2.5) with $f$ being the indicator of $C_{R^{2}, \varepsilon R}$ implies that

$$
E \mathrm{t}_{\tau_{\varepsilon, R}^{\prime}} \leq N E \int_{0}^{\tau_{R}} \kappa_{t} f\left(\mathrm{t}_{t}, x_{t}\right) d A_{t} \leq N \varepsilon^{d / p_{0}} R^{2}
$$

For an appropriate choice of $\varepsilon$ the last term is less than $(1 / 2) R^{2}$ and then

$$
P\left(\mathrm{t}_{\tau_{\varepsilon}^{\prime}} \geq R^{2}\right) \leq P\left(\mathrm{t}_{\tau_{\varepsilon, R}^{\prime}} \geq R^{2}\right) \leq 1 / 2 .
$$

Self-similarity implies that (2.20) holds with $T=\varepsilon^{-2}$ if $x=0$.

Estimate (2.23) below in case $b$ is bounded was the starting point for the theory of time homogeneous controlled diffusion processes.

Lemma 2.12. Assume (2.19) and (2.2). Then

$$
E \int_{0}^{\tau_{R}^{\prime}} \operatorname{tr} a_{t} d A_{t} \leq \delta^{-1} E \mathrm{t}_{\tau_{R}^{\prime}} \leq N\left(\delta, d, p_{0}, \bar{H}\right) R^{2},
$$


and for any Borel nonnegative $f(x)$

$$
E \int_{0}^{\tau_{R}^{\prime}} \kappa_{t} f\left(x_{t}\right) d A_{t} \leq N\left(d, p_{0}, p, \bar{H}, \delta\right) R^{2-d / p}\|f\|_{L_{p}\left(\mathbb{R}^{d}\right)} .
$$

In particular (as $p=d, q=\infty$ in $\kappa_{t}$ ),

$$
E \int_{0}^{\tau_{R}^{\prime}}\left(\operatorname{det} a_{t}\right)^{1 / d} f\left(x_{t}\right) d A_{t} \leq N\left(d, p_{0}, \bar{H}, \delta\right) R\|f\|_{L_{d}\left(\mathbb{R}^{d}\right)} .
$$

Proof. The first inequality in (2.21) is obvious. To prove the second one observe that with $T$ from Corollary 2.11

$$
E \mathrm{t}_{\tau_{R}^{\prime}} \leq \sum_{k=1}^{\infty} k T R^{2} P\left((k-1) T R^{2} \leq \mathrm{t}_{\tau_{R}^{\prime}}<k T R^{2}\right)
$$

For the event $\left\{(k-1) T R^{2} \leq \mathrm{t}_{\tau_{R}^{\prime}}<k T R^{2}\right\}$ to realize, the first coordinate of the process $\left(\mathrm{t}_{t}, x_{t}\right)$ should experience $k-1$ moves from $i T R^{2}$ to $(i+1) T R^{2}$ before $x_{t}$ exits from $B_{R}$. According to a "conditional" version of Corollary 2.11 the conditional probability of each such move given the past is less than $1 / 2$. Therefore,

$$
E \mathrm{t}_{\tau_{R}^{\prime}} \leq \sum_{k=1}^{\infty} k T R^{2} 2^{-k+1}
$$

and this yields the second inequality in (2.21).

To prove $(2.22)$ observe that for $k=1,2, \ldots, \Delta_{k}=\left[(k-1) T R^{2}, k T R^{2}\right)$,

$$
f_{k}(t, x)=I_{\Delta_{k}}(t) f(x)
$$

and $\gamma_{k}$ defined as the first time $\mathrm{t}_{t}$ hits $k T R^{2}$, according to (2.3) with $c=0$ on the set $\tau_{R}^{\prime} \geq \gamma_{k-1}$ we have

$$
\begin{aligned}
& E\left(\int_{\gamma_{k-1}}^{\gamma_{k} \wedge \tau_{R}^{\prime}} \kappa_{t} f\left(x_{t}\right) d A_{t} \mid \mathcal{F}_{\gamma_{k-1}}\right)=E\left(\int_{\gamma_{k-1}}^{\gamma_{k} \wedge \tau_{R}^{\prime}} \kappa_{t} f_{k}\left(\mathrm{t}_{t}, x_{t}\right) d A_{t} \mid \mathcal{F}_{\gamma_{k-1}}\right) \\
& \leq N\left(R^{2}+\|h\|_{L_{p_{0}, q_{0}}\left(\Delta_{k} \times B_{R}\right)}^{2 q_{0}}\right)^{d /(2 p)} R^{2 / q}\|f\|_{L_{p}\left(\mathbb{R}^{d}\right)} \leq N R^{2-d / p}\|f\|_{L_{p}\left(\mathbb{R}^{d}\right)} .
\end{aligned}
$$

It follows that

$$
\begin{gathered}
E \int_{0}^{\tau_{R}^{\prime}} \kappa_{t} f\left(x_{t}\right) d A_{t}=\sum_{k=1}^{\infty} E \int_{\gamma_{k-1} \wedge \tau_{R}^{\prime}}^{\gamma_{k} \wedge \tau_{R}^{\prime}} \kappa_{t} f\left(x_{t}\right) d A_{t} \\
=\sum_{k=1}^{\infty} E I_{\tau_{R}^{\prime} \geq \gamma_{k-1}} \int_{\gamma_{k-1}}^{\gamma_{k} \wedge \tau_{R}^{\prime}} \kappa_{t} f\left(x_{t}\right) d A_{t} \\
\leq N R^{2-d / p}\|f\|_{L_{p}\left(\mathbb{R}^{d}\right)} \sum_{k=1}^{\infty} P\left(\tau_{R}^{\prime} \geq \gamma_{k-1}\right) .
\end{gathered}
$$

As we have seen above, each of the probabilities in the last sum is less than $2^{-k+1}$ and this proves the lemma. 


\section{Green's FunCtions}

In this section we make one step closer to stochastic Itô equations and restrict the class of processes from Section 2. We suppose that

$$
A_{t}=t, \quad r_{t} \equiv 1, \quad \delta^{-1}|\lambda|^{2} \geq\left(a_{t} \lambda, \lambda\right) \geq \delta|\lambda|^{2},
$$

Assumption 2.1 is satisfied and also suppose that we are given $p$ and $q$ satisfying (2.16).

Here is a straightforward consequence of (2.17).

Theorem 3.1. There exists a constant $N=N\left(\delta, d, \bar{H}, p, q, p_{0}, W\right)$ such that for any $\lambda>0$ there exists a nonnegative Borel function $G_{\lambda}(t, x)$ (Green's function of (t., $x$.$) ) on \mathbb{R}^{d+1}$ such that $G_{\lambda}(t, x)=0$ for $t \leq 0$ and for any Borel nonnegative $f$ given on $\mathbb{R}^{d+1}$ we have

$$
\begin{gathered}
E \int_{0}^{\infty} e^{-\lambda t} f\left(\mathrm{t}_{t}, x_{t}\right) d t=\int_{\mathbb{R}^{d+1}} f(t, x) G_{\lambda}(t, x) d x d t, \\
\left\|\Phi_{\lambda}^{\nu-1} G_{\lambda}\right\|_{L_{p, q}^{\prime}} \leq N \lambda^{-\nu-d /(2 p)}
\end{gathered}
$$

where we use the notation

$$
\begin{aligned}
& \|u\|_{L_{p, q}^{\prime}}=\left(\int_{\mathbb{R}}\left(\int_{\mathbb{R}^{d}}|u(t . x)|^{p^{\prime}} d x\right)^{q^{\prime} / p^{\prime}} d t\right)^{1 / q^{\prime}} \quad \text { if } \quad p \geq q, \\
& \|u\|_{L_{p, q}^{\prime}}=\left(\int_{\mathbb{R}^{d}}\left(\int_{\mathbb{R}}|u(t . x)|^{q^{\prime}} d t\right)^{p^{\prime} / q^{\prime}} d x\right)^{1 / p^{\prime}} \quad \text { if } \quad p<q,
\end{aligned}
$$

and $p^{\prime}=p /(p-1), q^{\prime}=q /(q-1)$.

The highest power of pure $(p=q=d+1)$ summability of $G_{\lambda}$ guaranteed by this theorem is $1+1 / d$. It turns out that, actually, $G_{\lambda}$ is summable to a higher power. The proof of this is based on the parabolic version of Gehring's lemma from [6].

Introduce $\mathbb{Q}$ as the set of cylinders $C_{R}(t, x), R>0, t \geq 0, x \in \mathbb{R}^{d}$. For $Q=C_{R}(t, x) \in \mathbb{Q}$ let $2 Q=C_{2 R}(t, x)$. If $Q \in \mathbb{Q}$ and $Q=C_{R}(t, x)$, we call $R$ the radius of $Q$.

Theorem 3.2. There exist $d_{0} \in(1, d)$ and a constant $N$, depending only on $d, \delta, \bar{H}$, and $W$, such that for any $Q \in \mathbb{Q}$ of radius $R \leq 1 / 2$ and $p \geq d_{0}+1$, we have

$$
\left\|G_{1}\right\|_{L_{p /(p-1)}(Q)} \leq N R^{-(d+2) / p}\left\|G_{1}\right\|_{L_{1}(2 Q)},
$$

which is equivalently rewritten as

$$
\left(f_{Q} G_{1}^{p /(p-1)} d x d t\right)^{(p-1) / p} \leq N f_{2 Q} G_{1} d x d t .
$$

Proof. We basically follow the idea in [5]. Take $R \in(0,1 / 2], Q \in \mathbb{Q}$ of radius $R$, and define recursively

$$
\begin{gathered}
\gamma^{1}=\inf \left\{t \geq 0:\left(\mathrm{t}_{t}, x_{t}\right) \in \bar{Q}\right\}, \quad \tau^{1}=\inf \left\{t \geq \gamma^{1}:\left(\mathrm{t}_{t}, x_{t}\right) \notin 2 Q\right\}, \\
\gamma^{n+1}=\inf \left\{t \geq \tau^{n}:\left(\mathrm{t}_{t}, x_{t}\right) \in \bar{Q}\right\}, \quad \tau^{n+1}=\inf \left\{t \geq \gamma^{n+1}:\left(\mathrm{t}_{t}, x_{t}\right) \notin 2 Q\right\} .
\end{gathered}
$$


Then for any nonnegative Borel $f$ vanishing outside $Q$ with $\|f\|_{L_{d+1}(Q)}=1$ we have

$$
\begin{gathered}
\int_{Q} f G_{1}(t, x) d x d t=E \int_{0}^{\infty} e^{-t} f\left(t, x_{t}\right) d t \\
=\sum_{n=1}^{\infty} E e^{-\gamma^{n}} E\left(\int_{\gamma^{n}}^{\tau^{n}} e^{-\left(t-\gamma^{n}\right)} f\left(t, x_{t}\right) d t \mid \mathcal{F}_{\gamma^{n}}\right) .
\end{gathered}
$$

Next we use the conditional version of (2.5) to see that the conditional expectations above are less than $N R^{d /(d+1)}$. After that we use the conditional version of Corollary 2.6 to get that

$$
R^{2} \leq N E\left(\int_{\gamma^{n}}^{\tau^{n}} e^{-\left(t-\gamma^{n}\right)} d t \mid \mathcal{F}_{\gamma^{n}}\right)
$$

Then we obtain

$$
\begin{gathered}
\int_{Q} f G_{1}(t, x) d x d t \leq N R^{-(d+2) /(d+1)} \sum_{n=1}^{\infty} E e^{-\gamma^{n}} E\left(\int_{\gamma^{n}}^{\tau^{n}} e^{-\left(t-\gamma^{n}\right)} d t \mid \mathcal{F}_{\gamma^{n}}\right) \\
=N R^{-(d+2) /(d+1)} \sum_{n=1}^{\infty} E \int_{\gamma^{n}}^{\tau^{n}} e^{-t} d t \\
\leq N R^{-(d+2) /(d+1)} E \int_{0}^{\infty} e^{-t} I_{2 Q}\left(t, x_{t}\right) d t=N R^{-(d+2) /(d+1)} \int_{2 Q} G_{1}(t, x) d x d t .
\end{gathered}
$$

The arbitrariness of $f$ implies that

$$
\left(f_{Q} G_{1}^{(d+1) / d}(t, x) d x d t\right)^{d /(d+1)} \leq N f_{2 Q} G_{1}(t, x) d x d t .
$$

Now the assertion of the theorem for $p=d_{0}$ follows directly from the corrected version of the famous Gehring's lemma stated as Proposition 1.3 in [6]. For larger $p$ it suffices to use Hölder's inequality. The theorem is proved.

Remark 3.1. The result of Theorem 3.2 in case $x_{t}$ is a solution of a stochastic equation with deterministic $a(t, x)$ and $b \equiv 0$ can be found in the PDE terms in Remark 1 of [4], where it is given without proof. Complete proof for bounded $b(t, x)$ can be extracted from [2] dealing with viscosity solutions of fully nonlinear parabolic equations. With little effort this proof can be used to obtain the result of Theorem 3.2 if $b$ is bounded. Our $b$ is in $L_{p, q}$.

Similar improvement of integrability occurs for the Green's function of $x_{t}$ rather than $\left(t, x_{t}\right)$. Here is a straightforward consequence of $(2.18)$.

Theorem 3.3. Let $p \in[d, \infty)$. Then for any $\lambda>0$ there exists a nonnegative Borel function $g_{\lambda}(x)$ (Green's function of $x$.) on $\mathbb{R}^{d}$ such that for any Borel nonnegative $f$ given on $\mathbb{R}^{d}$ we have

$$
E \int_{0}^{\infty} e^{-\lambda t} f\left(x_{t}\right) d t=\int_{\mathbb{R}^{d}} f(x) g_{\lambda}(x) d x
$$




$$
\left\|\Psi_{\lambda}^{-d / p} g_{\lambda}\right\|_{L_{p^{\prime}}} \leq N \lambda^{-1+d /(2 p)},
$$

where $\Psi_{\lambda}(x)=\exp (-\sqrt{\lambda}|x| \theta / 32), p^{\prime}=p /(p-1)$, and $N$ depends only on $\delta$, $d, \bar{H}, p, p_{0}, W$.

According to this theorem this Green's function is summable to the power $d /(d-1)$. Again it turns out that this power can be increased. If $B$ is an open ball in $\mathbb{R}^{d}$ by $2 B$ we denote the concentric open ball of twice the radius of $B$.

Theorem 3.4. There exist $d_{0} \in(1, d)$ and a constant $N$, depending only on $d, \delta, \bar{H}$, and $W$, such that for any ball $B$ of radius $R \leq 1 / 2$ and $p \geq d_{0}$, we have

$$
\left\|g_{1}\right\|_{L_{p /(p-1)}(B)} \leq N R^{-d / p}\left\|g_{1}\right\|_{L_{1}(2 B)},
$$

which is equivalently rewritten as

$$
\left(f_{B} g_{1}^{p /(p-1)} d x\right)^{(p-1) / p} \leq N f_{2 B} g_{1} d x
$$

Proof. We again follow the idea in [5]. Take $R \in(0,1 / 2]$, an open ball $B$ of radius $R$, and define recursively

$$
\begin{gathered}
\gamma^{1}=\inf \left\{t \geq 0: x_{t} \in \bar{B}\right\}, \quad \tau^{1}=\inf \left\{t \geq \gamma^{1}: x_{t} \notin 2 B\right\}, \\
\gamma^{n+1}=\inf \left\{t \geq \tau^{n}: x_{t} \in \bar{B}\right\}, \quad \tau^{n+1}=\inf \left\{t \geq \gamma^{n+1}: x_{t} \notin 2 B\right\} .
\end{gathered}
$$

Then for any nonnegative Borel $f$ vanishing outside $B$ with $\|f\|_{L_{d}(B)}=1$ we have

$$
\begin{gathered}
\int_{B} f g_{1}(x) d x=E \int_{0}^{\infty} e^{-t} f\left(x_{t}\right) d t \\
=\sum_{n=1}^{\infty} E e^{-\gamma^{n}} E\left(\int_{\gamma^{n}}^{\tau^{n}} e^{-\left(t-\gamma^{n}\right)} f\left(x_{t}\right) d t \mid \mathcal{F}_{\gamma^{n}}\right) .
\end{gathered}
$$

Next we use the conditional version of (2.23) to see that the conditional expectations above are less than $N R$. After that we use the conditional version of Corollary 2.6 to get that

$$
R^{2} \leq N E\left(\int_{\gamma^{n}}^{\tau^{n}} e^{-\left(t-\gamma^{n}\right)} d t \mid \mathcal{F}_{\gamma^{n}}\right) .
$$

Then we obtain

$$
\begin{gathered}
\int_{B} f g_{1}(x) d x \leq N R^{-1} \sum_{n=1}^{\infty} E e^{-\gamma^{n}} E\left(\int_{\gamma^{n}}^{\tau^{n}} e^{-\left(t-\gamma^{n}\right)} d t \mid \mathcal{F}_{\gamma^{n}}\right) \\
=N R^{-1} \sum_{n=1}^{\infty} E \int_{\gamma^{n}}^{\tau^{n}} e^{-t} d t \\
\leq N R^{-1} E \int_{0}^{\infty} e^{-t} I_{2 B}\left(x_{t}\right) d t=N R^{-1} \int_{2 B} g_{1}(x) d x .
\end{gathered}
$$


The arbitrariness of $f$ implies that

$$
\left(f_{B} g_{1}^{d /(d-1)}(x) d x\right)^{(d-1) / d} \leq N f_{2 B} g_{1}(x) d x
$$

and again it only remains to use Gehring's lemma in case $p=d$. For larger $p$ it suffices to use Hölder's inequality. The theorem is proved.

Below by $d_{0}$ we mean the largest of $d_{0}$ 's from Theorems 3.2 and 3.4 and first present improved parabolic Aleksandrov estimates by following the interpolation arguments in Nazarov [15].

Lemma 3.5. Suppose that

$$
p, q \in[1, \infty], \quad \frac{d_{0}}{p}+\frac{1}{q}=1
$$

Then for any Borel $f(t, x) \geq 0$ vanishing outside a $Q \in \mathbb{Q}$ of radius $1 / 2$

$$
I:=E \int_{0}^{\infty} e^{-t} f\left(t, x_{t}\right) d t \leq N\|f\|_{L_{p, q}},
$$

where $N=N\left(\delta, d, \bar{H}, p, p_{0}, W\right)$.

Proof. If $p=d_{0}+1$, then $q=d_{0}+1$ and (3.6) follows from (3.2) and the fact that, obviously, $\left\|G_{1}\right\|_{L_{1,1}}=1$. In other terms, for any Borel $f(t, x) \geq 0$ vanishing outside $Q=\left(t_{0}, t_{t}\right) \times B_{R}(y)$

$$
E \int_{0}^{\infty} e^{-t} f\left(t, x_{t}\right) d t=\int_{Q} G_{1}(t, x) f(t, x) d x d t \leq N\|f\|_{L_{d_{0}+1, d_{0}+1}} .
$$

If $p=d_{0}$ and $q=\infty$ estimate (3.6) follows from (3.4) since

$$
\begin{aligned}
I \leq & E \int_{0}^{\infty} e^{-t} \sup _{s \geq 0} f\left(s, x_{t}\right) d t=\int_{\mathbb{R}^{d}} g_{1}(x) \sup _{s \geq 0} f(s, x) d x \\
& \leq N\left(\int_{\mathbb{R}^{d}} \sup _{s \geq 0} f^{d_{0}}(s, x) d x\right)^{1 / d_{0}}=N\|f\|_{L_{d_{0}, \infty}} .
\end{aligned}
$$

If $p=\infty$ and $q=1$

$$
I \leq \int_{0}^{\infty} \sup _{x} f(t, x) d t=\|f\|_{L_{\infty, 1}}
$$

We will use these facts in an interpolation argument. In case $\infty>p>$ $d_{0}+1$ we have $p>q$ and set $\beta=p /\left(d_{0}+1\right)$ and $\alpha=\beta /(\beta-1)$. Take a nonnegative $g(t)$ such that $(f(t, x) g(t)) / g(t)=f(t, x)(0 / 0=0)$ and use Hölder's inequality to conclude that $I \leq I_{1} I_{2}$, where

$$
\begin{gathered}
I_{1}=\left(\int_{0}^{\infty} g^{-\alpha}(t) d t\right)^{1 / \alpha} \\
I_{2}=\left(E \int_{0}^{\infty} e^{-t} g^{\beta}(t) f^{\beta}\left(t, x_{t}\right) d t\right)^{1 / \beta} \\
\leq N\left(\int_{0}^{\infty} g^{\left(d_{0}+1\right) \beta}(t)\left(\int_{\mathbb{R}^{d}} f^{\left(d_{0}+1\right) \beta}(t, x) d x\right) d t\right)^{1 /\left(d_{0} \beta+\beta\right)} .
\end{gathered}
$$


For $g$ found from

$$
g^{-\alpha}(t)=g^{\left(d_{0}+1\right) \beta}(t) \int_{\mathbb{R}^{d}} f^{\left(d_{0}+1\right) \beta}(t, x) d x
$$

we get (3.6) and this takes care of the case that $\infty>p>d_{0}+1$.

If $\infty>q>d_{0}+1$ we have $p<q$ and set $\beta=q /\left(q-d_{0}-1\right)$ and $\alpha=$ $\beta /(\beta-1)$. Take a nonnegative $g(x)$ such that $(f(t, x) g(x)) / g(x)=f(t, x)$ $(0 / 0=0)$ and use Hölder's inequality to conclude that $I \leq I_{1} I_{2}$, where

$$
\begin{gathered}
I_{1}=\left(E \int_{0}^{\infty} e^{-t} g^{-\beta}\left(x_{t}\right) d t\right)^{1 / \beta} \leq N\left(\int_{\mathbb{R}^{d}} g^{-d_{0} \beta}(x) d x\right)^{1 /\left(d_{0} \beta\right)}, \\
I_{2}=\left(E \int_{0}^{\infty} e^{-t} g^{\alpha}\left(x_{t}\right) f^{\alpha}\left(t, x_{t}\right) d t\right)^{1 / \alpha} \\
\leq N\left(\int_{\mathbb{R}^{d}} g^{\left(d_{0}+1\right) \alpha}(x)\left(\int_{0}^{\infty} f^{\left(d_{0}+1\right) \alpha}(t, x) d t\right) d x\right)^{1 /\left(\alpha d_{0}+\alpha\right)} .
\end{gathered}
$$

For $g$ found from

$$
g^{-d_{0} \beta}(x)=g^{\left(d_{0}+1\right) \alpha}(x) \int_{0}^{\infty} f^{\left(d_{0}+1\right) \alpha}(t, x) d t
$$

we get (3.6) and this proves the lemma.

Using this lemma instead of (2.5) and just repeating the proof of Lemma 2.8 we come to a natural counterpart of the latter and then by literally repeating the proof of Theorem 2.9 we come to the following result, that is a version of Theorem 4.1 of Nazarov [15] in which $d / p+1 / q \leq 1$ that is stronger than ours, but in which the assumption on $h$ is weaker. A proper probabilistic version of Theorem 4.1 of Nazarov [15] is found in [8]. Set

$$
\mathbb{R}_{+}^{d+1}=\{t \geq 0\} \cap \mathbb{R}^{d+1} .
$$

Theorem 3.6. Suppose

$$
p, q \in[1, \infty], \quad \nu:=1-\frac{d_{0}}{p}-\frac{1}{q} \geq 0 .
$$

Then there is $N=N\left(\delta, d, \bar{H}, p, q, p_{0}, W\right)$ such that for any $\lambda>0$ and Borel nonnegative $f$ we have

$$
E \int_{0}^{\infty} e^{-\lambda t} f\left(t, x_{t}\right) d t \leq N \lambda^{-\nu+\left(d-2 d_{0}\right) /(2 p)}\left\|\Phi_{\lambda}^{1-\nu} f\right\|_{L_{p, q}\left(\mathbb{R}_{+}^{d+1}\right)},
$$

where $\Phi_{\lambda}(t, x)=\exp (-\sqrt{\lambda}(|x|+\sqrt{t}) \theta / 32)$. In particular, if $f$ is independent of $t, p \geq d_{0}$, and $q=\infty$

$$
E \int_{0}^{\infty} e^{-\lambda t} f\left(x_{t}\right) d t \leq N \lambda^{-1+d /(2 p)}\left\|\bar{\Phi}_{\lambda}^{d_{0} / p} f\right\|_{L_{p}\left(\mathbb{R}^{d}\right)},
$$

where $\bar{\Phi}_{\lambda}(x)=\exp (-\sqrt{\lambda}|x| \theta / 32)$. 
Theorem 3.7. Assume that (3.7) holds. Then for any $n=1,2, \ldots$, nonnegative Borel $f$ on $\mathbb{R}_{+}^{d+1}$, and $T \in(0, \infty)$ we have

$$
E\left[\int_{0}^{T} f\left(t, x_{t}\right) d t\right]^{n} \leq n ! N^{n} T^{n \chi}\left\|\Phi_{1 / T}^{(1-\nu) / n} f\right\|_{L_{p, q}\left(\mathbb{R}_{+}^{d+1}\right)}^{n},
$$

where $N=N\left(\delta, d, \bar{H}, p, q, p_{0}, W\right)$ and $\chi=\nu+\left(2 d_{0}-d\right) /(2 p)$.

Proof. The proof of this theorem proceeds by induction on $n$ and is achieved by almost literally repeating the proof of Theorem 2.6 of [11]. The induction hypothesis is that for all $(t, x) \in \mathbb{R}_{+}^{d+1}$ and $\kappa \in[0,1 / n]$

$$
E\left[\int_{0}^{T} f\left(t+s, x+x_{s}\right) d s\right]^{n} \leq n ! N^{n} T^{n \chi} \Phi_{1 / T}^{(\nu-1) \kappa n}(t, x)\left\|\Phi_{1 / T}^{(1-\nu) \kappa} f\right\|_{L_{p, q}\left(\mathbb{R}_{+}^{d+1}\right)}^{n} .
$$

We will discuss in detail only the case of $n=1$. In that case observe that, for any $\lambda>0$

$$
I:=E \int_{0}^{T} f\left(t+s, x+x_{s}\right) d s \leq e^{\lambda T} E \int_{0}^{\infty} e^{-\lambda s} f\left(t+s, x+x_{s}\right) d s .
$$

It follows from Theorem 3.6 that for any $\kappa \in[0,1]$

$$
\begin{gathered}
e^{-\lambda T} I \leq N \lambda^{-\chi}\left\|f(t+\cdot, x+\cdot) \Phi_{\lambda}^{(1-\nu) \kappa}\right\|_{L_{p, q}\left(\mathbb{R}_{+}^{d+1}\right)} \\
\leq N \lambda^{-\chi} \Phi_{\lambda}^{(\nu-1) \kappa}(t, x)\left\|f \Phi_{\lambda}^{(1-\nu) \kappa}\right\|_{L_{p, q}\left(\mathbb{R}_{+}^{d+1}\right)},
\end{gathered}
$$

where the last inequality is due to the fact that, for $(s, y) \in \mathbb{R}_{+}^{d+1}, \Phi_{\lambda}(s, y) \leq$ $\Phi_{\lambda}(t+s, x+y) \Phi_{\lambda}^{-1}(t, x)$. For $\lambda=1 / T$ we get (3.10) with $n=1$. The theorem is proved.

Next theorem improves estimate (2.5) in what concerns the restrictions on $p, q$.

Theorem 3.8. Assume that (3.7) holds with $\nu=0$. Then for any $R>0$ and Borel nonnegative $f$ given on $C_{R}$, we have

$$
E \int_{0}^{\tau_{R}} f\left(t, x_{t}\right) d t \leq N R^{\left(2 d_{0}-d\right) / p}\|f\|_{L_{p, q}\left(C_{R}\right)}
$$

where $N=N\left(\delta, d, \bar{H}, p, p_{0}, W\right)$.

Proof. Self-similar transformations show that we may assume that $R=1$. Also we may assume that $f$ is bounded and is zero outside $C_{1}$. In that case denote by $\mathfrak{M}$ the set of stopping times $\gamma \leq \tau:=\tau_{1}$, and set

$$
u_{\gamma}=E\left[\int_{\gamma}^{\tau} f\left(t, x_{t}\right) d t \mid \mathcal{F}_{\gamma}\right], \quad \bar{u}=\underset{\gamma \in \mathfrak{M}}{\operatorname{esssup}} u_{\gamma} .
$$

Observe that for any $\omega$ and $\lambda>0$ it holds that

$$
\int_{\gamma}^{\tau} f\left(t, x_{t}\right) d t=\int_{\gamma}^{\tau} e^{-\lambda(t-\gamma)} f\left(t . x_{t}\right) d t
$$




$$
+\lambda \int_{0}^{\infty} e^{-\lambda(t-\gamma)} I_{\gamma \leq t<\tau}\left[\int_{t}^{\tau} f\left(s, x_{s}\right) d s\right] d t .
$$

By the conditional version of Theorem 3.6 (a.s.)

Hence,

$$
\begin{gathered}
E\left[\int_{\gamma}^{\tau} e^{-\lambda(t-\gamma)} f\left(t, x_{t}\right) d t \mid \mathcal{F}_{\gamma}\right] \\
\leq E\left[\int_{\gamma}^{\infty} e^{-\lambda(t-\gamma)} f\left(t, x_{t}\right) d t \mid \mathcal{F}_{\gamma}\right] \leq N \lambda^{\left(d-2 d_{0}\right) /(2 p)}\|f\|_{L_{p, q}} .
\end{gathered}
$$

$$
\begin{gathered}
u_{\gamma} \leq N \lambda^{\left(d-2 d_{0}\right) /(2 p)}\|f\|_{L_{p, q}} \\
+\lambda E\left[\int_{\gamma}^{\infty} e^{-\lambda(t-\gamma)} I_{\gamma \leq t<\tau} E\left[\int_{t}^{\tau} f\left(x_{s}\right) d s \mid \mathcal{F}_{t}\right] d t \mid \mathcal{F}_{\gamma}\right],
\end{gathered}
$$

where the last term is dominated by

$$
\lambda \bar{u} E\left[\int_{\gamma}^{\infty} e^{-\lambda(t-\gamma)} I_{\gamma \leq t<\tau} d t \mid \mathcal{F}_{\gamma}\right] \leq \lambda \bar{u} E\left[\int_{\gamma}^{\tau} d t \mid \mathcal{F}_{\gamma}\right] \leq N_{1} \lambda \bar{u}
$$

(a.s.), where the last inequality follows from the conditional version of (2.5). Thus, (a.s.)

$$
u_{\gamma} \leq N \lambda^{\left(d-2 d_{0}\right) /(2 p)}\|f\|_{L_{p, q}}+N_{1} \lambda \bar{u} .
$$

Since $\gamma$ is arbitrary within $\mathfrak{M}$,

$$
\bar{u} \leq N \lambda^{\left(d-2 d_{0}\right) /(2 p)}\|f\|_{L_{p, q}}+N_{1} \lambda \bar{u}
$$

(a.s.), and since $\bar{u}<\infty$ ( $f$ is bounded $)$, by taking $\lambda=1 /\left(2 N_{1}\right)$, we arrive at

$$
\bar{u} \leq N\|f\|_{L_{p, q}} \text {. }
$$

The theorem is proved.

Remark 3.2. Equation (3.12) implies that $d_{0} \geq d / 2$. Of course, the example of the Wiener process with no drift shows more than that, actually, $d_{0}>d / 2$.

Theorem 3.8 allows us to prove Itô's formula for functions $u \in W_{p, q}^{1,2}(Q)$, where $Q$ is a domain in $\mathbb{R}^{d+1}$ and

$$
W_{p, q}^{1,2}(Q)=\left\{v: v, \partial_{t} v, D v, D^{2} v \in L_{p, q}(Q)\right\}
$$

with norm introduced in a natural way. Before, the formula was known only for (smooth, Itô, and) $W_{d+1}^{1,2}$-functions and processes with bounded drifts or for $W_{d_{0}}^{2}$-functions in case the drift of the process is dominated by $h\left(x_{t}\right)$ with $h \in L_{d}($ see $[11])$.

The following extends Theorem 2.10.1 of [9].

Theorem 3.9. Assume that (3.7) holds with $\nu=0$ and $p<\infty, q<\infty$. Let $Q$ be a bounded domain in $\mathbb{R}^{d+1}, 0 \in Q, b$ be bounded, and $u \in W_{p, q}^{1,2}(Q) \cap$ $C(\bar{Q})$. Then, for $\tau$ defined as the first exit time of $\left(t, x_{t}\right)$ from $Q$ and for all $t \geq 0$,

$$
u\left(t \wedge \tau, x_{t \wedge \tau}\right)=u(0,0)+\int_{0}^{t \wedge \tau} D_{i} u\left(s, x_{s}\right) d m_{s}^{i}
$$




$$
+\int_{0}^{t \wedge \tau}\left[\partial_{t} u\left(s, x_{s}\right)+a_{s}^{i j} D_{i j} u\left(s, x_{s}\right)+b_{s}^{i} D_{i} u\left(s, x_{s}\right)\right] d s
$$

and the stochastic integral above is a square-integrable martingale.

Proof. First assume that $u$ is smooth and its derivatives are bounded. Then (3.13) holds by Itô's formula and, moreover,

$$
\begin{gathered}
E \int_{0}^{\tau}\left|D u\left(s, x_{s}\right)\right|^{2} d s \leq N E\left(\int_{0}^{\tau} D_{i} u\left(s, x_{s}\right) d m_{s}^{i}\right)^{2} \\
=N E\left(u\left(\tau, x_{\tau}\right)-u(0,0)-\int_{0}^{\tau}\left[\partial_{t} u\left(s, x_{s}\right)+a_{s}^{i j} D_{i j} u\left(s, x_{s}\right)+b_{s}^{i} D_{i} u\left(s, x_{s}\right)\right] d s\right)^{2} \\
\leq N \sup _{\bar{Q}}|u|+N E\left(\int_{0}^{T} I_{Q}\left(\left|\partial_{t} u\right|+|D u|+\left|D^{2} u\right|\right)\left(s, x_{s}\right) d s\right)^{2},
\end{gathered}
$$

where $T$ is the size of $Q$ in the $t$-direction. In light of Theorem 3.7 we conclude that

$$
E \int_{0}^{\tau}\left|D u\left(s, x_{s}\right)\right|^{2} d s \leq N \sup _{\bar{Q}}|u|+N T^{\left(2 d_{0}-d\right) / p}\left\|\partial_{t} u, D u, D^{2} u\right\|_{L_{p, q}(Q)},
$$

where $N$ are independent of $u$ and $Q$. Owing to Fatou's theorem, this estimate is also true for those $u \in W_{p, q}^{1,2}(Q) \cap C(\bar{Q})$ that can be approximated uniformly and in the $W_{p, q}^{1,2}(Q)$-norm by smooth functions with bounded derivatives (recall that $p<\infty, q<\infty$ ). For our $u$ there is no guarantee that such approximation is possible. However, mollifiers do such approximations in any subdomain $Q^{\prime} \subset \bar{Q}^{\prime} \subset Q$. Hence, (3.14) holds for our $u$ if we replace $Q$ by $Q^{\prime}$ (containing $(0,0)$ ). Setting $Q^{\prime} \uparrow Q$ proves (3.14) in the generals case and proves the last assertion of the theorem.

After that (3.13) with $Q^{\prime}$ in place of $Q$ is proved by routine approximation of $u$ by smooth functions. Setting $Q^{\prime} \uparrow Q$ finally proves (3.13). The theorem is proved.

By using our Theorems 2.5 and 3.8 (with $p=d+1$ ) instead of Theorems 2.10 and 4.1 of [10] and arguing as in the proof of Theorem 4.4 of [10] we come to the following. Theorem 3.10 originated in [12] in case $b$ is bounded.

Theorem 3.10. For any $\kappa \in(0,1)$ there is a function $q(\gamma), \gamma \in(0,1)$, depending only on $\kappa, \delta, d, \bar{H}, p_{0}, W$, and, naturally, on $\gamma$, such that for any $R \in(0, \infty), x \in B_{\kappa R}$, and closed $\Gamma \subset C_{R}$ satisfying $|\Gamma| \geq \gamma\left|C_{R}\right|$ we have

$$
P\left(\tau_{\Gamma}(x) \leq \tau_{R}(x)\right) \geq q(\gamma)
$$

where $\tau_{\Gamma}(x)$ is the first time the process $\left(t, x+x_{t}\right)$ hits $\Gamma$ and $\tau_{R}(x)$ is its first exit time from $C_{R}$. Furthermore, $q(\gamma) \rightarrow 1$ as $\gamma \uparrow 1$.

\section{The CASE Of Diffusion PROCESSES}

Fix a constant $\delta \in(0,1)$ and by $\mathbb{S}_{\delta}$ denote the set of $d \times d$-symmetric matrices whose eigenvalues are between $\delta$ and $\delta^{-1}$. In this section we impose the following. 
Assumption 4.1. (i) On $\mathbb{R}^{d+1}$ we are given Borel measurable $\sigma(t, x)$ and $b(t, x)$ with values in $\mathbb{S}_{\delta}$ and in $\mathbb{R}^{d}$ respectively.

(ii) We are given $p_{0}, q_{0} \in[1, \infty)$ satisfying (1.1) and a function $h(t, x)$ satisfying (2.1) for any $0<\tau \leq \rho^{2}$ and $(t, x) \in \mathbb{R}^{d+1}$, where $\bar{H}$ is a fixed constant and $W$ is a function satisfying $W(0+)=0, W(1)=1$.

(iii) We have $|b| \leq h$.

Let $\Omega$ be the set of $\mathbb{R}^{d+1}$-valued continuous function $\left(t_{0}+t, x_{t}\right), t_{0} \in \mathbb{R}$, defined for $t \in[0, \infty)$. For $\omega=\left\{\left(t_{0}+t, x_{t}\right), t \geq 0\right\}$, define $t_{t}(\omega)=t_{0}+t$, $x_{t}(\omega)=x_{t}$, and set $\mathcal{N}_{t}=\sigma\left(\left(\mathrm{t}_{s}, x_{s}\right), s \leq t\right), \mathcal{N}=\mathcal{N}_{\infty}$. In the following theorem which is Theorem 6.1 of [8] we use the terminology from [3].

Theorem 4.1. On $\mathbb{R}^{d+1}$ there exists a strong Markov process

$$
X=\left\{\left(\mathrm{t}_{t}, x_{t}\right), \infty, \mathcal{N}_{t}, P_{t, x}\right)
$$

such that the process

$$
X_{1}=\left\{\left(\mathrm{t}_{t}, x_{t}\right), \infty, \mathcal{N}_{t+}, P_{t, x}\right)
$$

is Markov and for any $(t, x) \in \mathbb{R}^{d+1}$ there exists a d-dimensional Wiener process $w_{t}, t \geq 0$, which is a Wiener process relative to $\overline{\mathcal{N}}_{t}$, where $\overline{\mathcal{N}}_{t}$ is the completion of $\mathcal{N}_{t}$ with respect to $P_{t, x}$, and such that with $P_{t, x}$-probability one, for all $s \geq 0, \mathrm{t}_{s}=t+s$ and

$$
x_{s}=x+\int_{0}^{s} \sigma\left(t+r, x_{r}\right) d w_{r}+\int_{0}^{s} b\left(t+r, x_{r}\right) d r .
$$

Remark 4.1. To be completely rigorous, to refer to [8] we should have $b \in$ $L_{p_{0}, q_{0}}$ (globally), and (2.1) is not needed. But with our $b$, owing to estimates (2.13) and (2.17), the arguments in [8] only simplify and do not require $b \in L_{p_{0}, q_{0}}$. Still it is worth saying that the author believes that under the conditions in [8] Harnack's inequality is true. Regarding the Hölder continuity of caloric functions in the same setting we have no guesses. The Hölder continuity seems to require some sort of self-similarity and the $L_{p_{0}, q_{0}}{ }^{-}$ norm is not preserved under such transformations if $p_{0}, q_{0}$ are subject to (1.1).

Theorem 4.2. For any $\lambda>0, p, q$ satisfying (3.7), and Borel nonnegative $f(t, x)$ and for

$$
R_{\lambda} f(t, x):=E_{t, x} \int_{0}^{\infty} e^{-\lambda s} f\left(t+s, x_{s}\right) d s .
$$

we have

$$
\left\|R_{\lambda} f\right\|_{L_{p, q}\left(\mathbb{R}_{+}^{d+1}\right)} \leq N \lambda^{-1}\|f\|_{L_{p, q}\left(\mathbb{R}_{+}^{d+1}\right)},
$$

where $N=N\left(\delta, d, \bar{H}, p, q, p_{0}, W\right)$.

Proof. Self-similar transformations allow us to concentrate on $\lambda=1$. By Theorem 3.6 (after appropriate shift of the origin in $\mathbb{R}^{d+1}$ ) we have

$$
R_{1} f(t, x) \leq N\left\|\Phi_{1}^{1-\nu} f(t+\cdot, x+\cdot)\right\|_{L_{p, q}\left(\mathbb{R}_{+}^{d+1}\right)} .
$$


Then, first assume that $p, q<\infty$. If $p \geq q$, (4.3) implies that

$$
\int_{\mathbb{R}^{d}}\left|R_{1} f(t, x)\right|^{p} d x \leq N \int_{\mathbb{R}^{d}}\left(\int_{0}^{\infty} F^{q / p}(t, s, x) d s\right)^{p / q} d x,
$$

where

$$
F(t, s, x)=\int_{\mathbb{R}^{d}} \Phi_{1}^{(1-\nu) p}(s, y) f^{p}(t+s, x+y) d y .
$$

By Minkowski's inequality the integral on the right in (4.4) is dominated by

$$
\left(\int_{0}^{\infty}\left(\int_{\mathbb{R}^{d}} F(t, s, x) d x\right)^{q / p} d s\right)^{p / q}
$$

where

$$
\begin{gathered}
\int_{\mathbb{R}^{d}} F(t, s, x) d x=\int_{\mathbb{R}^{d}} f^{p}(t+s, y) d y \int_{\mathbb{R}^{d}} \Phi_{1}^{(1-\nu) p}(s, y) d y \\
\leq N e^{-\mu \sqrt{s}} \int_{\mathbb{R}^{d}} f^{p}(t+s, y) d y
\end{gathered}
$$

with $\mu=\mu(\delta, p, q, \bar{H}, W)>0$. Below by $\mu$ we denote all such constants. It follows that

$$
\begin{gathered}
\int_{\mathbb{R}^{d}}\left|R_{1} f(t, x)\right|^{p} d x \leq N\left(\int_{0}^{\infty} e^{-\mu \sqrt{s}}\left(\int_{\mathbb{R}^{d}} f^{p}(t+s, y) d y\right)^{q / p} d s\right)^{p / q}, \\
\left\|R_{1} f\right\|_{L_{p, q}\left(\mathbb{R}_{+}^{d+1}\right)}^{q} \leq N\|f\|_{L_{p, q}\left(\mathbb{R}_{+}^{d+1}\right)^{q}}^{q}
\end{gathered}
$$

If $q \geq p$,

$$
\int_{0}^{\infty}\left|R_{1} f(t, x)\right|^{q} d t \leq N \int_{0}^{\infty}\left(\int_{\mathbb{R}^{d}} F^{p / q}(t, x, y) d y\right)^{q / p} d t
$$

where

$$
F(t, x, y)=\int_{0}^{\infty} \Phi_{1}^{(1-\nu) q}(s, y) f^{q}(t+s, x+y) d s .
$$

By Minkowski's inequality

$$
\left(\int_{0}^{\infty}\left|R_{1} f(t, x)\right|^{q} d t\right)^{p / q} \leq N \int_{\mathbb{R}^{d}}\left(\int_{0}^{\infty} F(t, x, y) d t\right)^{p / q} d y
$$

where

$$
\begin{gathered}
\int_{0}^{\infty} F(t, x, y) d t \leq \int_{0}^{\infty} f^{q}(s, x+y) d s \int_{0}^{\infty} \Phi_{1}^{(1-\nu) q}(s, y) d s \\
\leq N e^{-\mu|y|} \int_{0}^{\infty} f^{q}(s, x+y) d s .
\end{gathered}
$$

Hence,

$$
\begin{gathered}
\left(\int_{0}^{\infty}\left|R_{1} f(t, x)\right|^{q} d t\right)^{p / q} \leq N \int_{\mathbb{R}^{d}} e^{-\mu|y|}\left(\int_{0}^{\infty} f^{q}(s, x+y) d s\right)^{p / q} d y \\
\left\|R_{1} f\right\|_{L_{p, q}\left(\mathbb{R}_{+}^{d+1}\right)}^{p} \leq N\|f\|_{L_{p, q}\left(\mathbb{R}_{+}^{d+1}\right)}^{p}
\end{gathered}
$$

and we again come to (4.2). This takes care of the case that $p, q<\infty$. 
If $\nu=1$, then (4.2) follows from the fact that

$$
\sup _{\mathbb{R}_{+}^{d+1}} R_{1} f \leq \sup _{\mathbb{R}_{+}^{d+1}} f .
$$

If $\nu<1$ but $p=\infty$ (and $q<\infty$ ), then (4.2) follows from Minkowski's inequality and the fact that

$$
\sup _{x} R_{1} f(t, x) \leq \int_{0}^{\infty} e^{-s} \sup _{x} f(t+s, x) d t .
$$

If $\nu<1$ but $q=\infty$ (and $p<\infty$ ), then (4.3) implies that

$$
\sup _{t \geq 0}\left(R_{1} f(t, x)\right)^{p} \leq N \int_{\mathbb{R}^{d}} e^{-\mu|y|} \sup _{t \geq 0} f^{p}(t, x+y) d y .
$$

One sees that in this case (4.2) follows after using Minkowski's inequality once more. The theorem is proved.

\section{An APPliCATION TO FIRST-ORDER PARABOliC EQUATIONS}

We suppose that Assumption 4.1 is satisfied and take $p, q \in[1, \infty)$ such that

$$
\frac{d}{p}+\frac{1}{q}=1, \quad p>q_{0} d
$$

Theorem 5.1. Let $Q$ be a bounded domain in $\mathbb{R}^{d+1}$ with $0 \in Q$ and let $u \in W_{p, q}^{1,2}(Q) \cap C(\bar{Q})$ be such that $u \leq 0$ on $\partial^{\prime} Q$ and

$$
\partial_{t} u+b^{i} D_{i} u \leq 0
$$

in $Q$ (a.e.). Then $u(0) \leq 0$.

This theorem is an immediate consequence of the following result in which one need only send $\varepsilon \downarrow 0$ and take into account that $d / p+d^{2} q_{0} /\left(p p_{0}\right)=$ $q_{0} d / p<1$.

Lemma 5.2. Under the assumptions of Theorem 5.1 for any $\varepsilon>0$ we have

$$
u(0) \leq N \varepsilon^{-d / p-d^{2} q_{0} /\left(p p_{0}\right)}\|\varepsilon \Delta u\|_{L_{p, q}(Q)},
$$

where $N$ is independent of $\varepsilon$.

Proof. Take $\nu>0$ and let $x_{t}$ be a solution of the equation

$$
x_{t}=\varepsilon w_{t}+\int_{0}^{t} I_{(0, \nu)}\left(\left|b\left(s, x_{s}\right)\right|\right) b\left(s, x_{s}\right) d s
$$

on a probability space, where $w_{t}$ is a Wiener process. By Theorem 3.9 (with $t$ larger than the diameter of $Q$ )

$$
u(0) \leq-E \int_{0}^{\tau}\left[\partial_{t} u+(1 / 2) \varepsilon^{2} \Delta u+I_{(0, \nu)}(|b|) b^{i} D_{i} u\right]\left(s, x_{s}\right) d s .
$$

Here

$$
\partial_{t} u+(1 / 2) \varepsilon^{2} \Delta u+I_{(0, \nu)}(|b|) b^{i} D_{i} u \geq I_{(0, \nu)}(|b|)\left(\partial_{t} u+b^{i} D_{i} u\right)
$$




$$
+(1 / 2) \varepsilon^{2} \Delta u+I_{[\nu, \infty)}(|b|) \partial_{t} u \geq(1 / 2) \varepsilon^{2} \Delta u+I_{[\nu, \infty)}(|b|) \partial_{t} u .
$$

Also observe that with $I=\left(\delta^{i j}\right)$ we have

$$
|b| \leq N\left(\operatorname{det}\left(\varepsilon^{2} I / 2\right)^{1 / p_{0}} \varepsilon^{-2 d / p_{0}} h,\right.
$$

which by (2.5) implies that

$$
\begin{gathered}
E \int_{0}^{\tau} \varepsilon^{2 d / p}\left|(1 / 2) \varepsilon^{2} \Delta u+I_{[\nu, \infty)}(|b|) \partial_{t} u\right|\left(s, x_{s}\right) d s \\
\leq N\left(1+\varepsilon^{-2 d^{2} q_{0} /\left(p p_{0}\right)}\right)\left\|(1 / 2) \varepsilon^{2} \Delta u+I_{[\nu, \infty)}(|b|) \partial_{t} u\right\|_{L_{p, q}(Q)} .
\end{gathered}
$$

Hence,

$$
u(0) \leq N \varepsilon^{-2 d / p}\left(1+\varepsilon^{-2 d^{2} q_{0} /\left(p p_{0}\right)}\right)\left\|(1 / 2) \varepsilon^{2} \Delta u+I_{[\nu, \infty)}(|b|) \partial_{t} u\right\|_{L_{p, q}(Q)} .
$$

This is true with $N$ independent of $\varepsilon$ and $\nu$. By sending $\nu \rightarrow \infty$ we get (5.1) wit $\varepsilon^{2}$ in place of $\varepsilon$ which is irrelevant. The lemma is proved.

The result of Theorem 5.1 is close to be sharp in the following sense.

Example 5.1. Take $\varepsilon \in(0,1)$ and $p_{0}, q_{0} \in[1, \infty)$ such that $d / p_{0}+1 / q_{0}=1+\varepsilon$, $p_{0}<q_{0} d$ (say $q_{0} \geq 2$ ), and $p_{0} \geq d$. Then it turns out that there exists $b \in L_{p_{0}, q_{0}}, p, q \in[1, \infty)$ such that $d / p+1 / q=1$ and $p>q_{0} d$, a bounded domain $Q \subset \mathbb{R}^{d+1}$ such that $0 \in Q$, and a function $u \in W_{p, q}^{1,2}(Q) \cap C(\bar{Q})$ such that $u \leq 0$ on $\partial^{\prime} Q$ and

$$
\partial_{t} u+b^{i} D_{i} u \leq 0
$$

in $Q$ (a.e.), but $u(0)>0$.

To show this set

$$
\alpha=\frac{1-\varepsilon^{2}}{q_{0}}, \quad \beta=\frac{1-\varepsilon^{2}}{p_{0}} d
$$

and observe that, since $p_{0}<q_{0} d$, we have $\alpha<\beta$ and $1-\alpha>1-\beta$, so that there exists $p$ satisfying

$$
\frac{d}{1-\beta}>p>\frac{d}{1-\alpha} .
$$

Here the left-hand side is strictly bigger than $q_{0} d$ since

$$
q_{0}(1-\beta)=q_{0}\left(1-\frac{d}{p_{0}}+\frac{\varepsilon^{2}}{p_{0}} d\right)=q_{0}\left(\frac{1}{q_{0}}-\varepsilon+\frac{\varepsilon^{2}}{p_{0}} d\right)<1
$$

in light of $p_{0} \geq d$ and $\varepsilon \in(0,1)$. Therefore, we can choose $p$ satisfying (5.2) and such that $p>q_{0} d$ as required.

After that $q$ is also defined and we set

$$
\begin{gathered}
u(t, x)=2-\exp \left(|t|^{1-\alpha}+|x|^{1+\beta}\right), \quad Q=\{(t, x): u(t, x)>0\}, \\
b(t, x)=-\frac{1-\alpha}{1+\beta} \frac{1}{|t|^{\alpha}|x|^{\beta}} \frac{x}{|x|} \operatorname{sign} t .
\end{gathered}
$$

Since $\alpha q_{0}<1$ and $\beta p_{0}<d$, we have $b \in L_{p_{0}, q_{0}}(Q)$. Also the inequality $\alpha q<$ 1 , guaranteeing that $\partial_{t} u \in L_{p_{0}, q_{0}}(Q)$, is equivalent to the right inequality in (5.2), whereas $p(1-\beta)<d$, guaranteeing that $D^{2} u \in L_{p_{0}, q_{0}}(Q)$, is equivalent 
to the left inequality in (5.2). Hence, $u \in W_{p_{0}, q_{0}}^{1,2}(Q), u$ is also continuous, equals zero on the whole boundary of $Q, u(0)=1$, and, as is easy to see, $\partial_{t} u+b^{i} D_{i} u=0$ apart from the plane $t=0$.

Acknowledgment. The author is sincerely grateful to A.I. Nazarov for useful discussion of the results.

\section{REFERENCES}

[1] L. Beck, F. Flandoli, M. Gubinelli, and M. Maurelli, Stochastic ODEs and stochastic linear PDEs with critical drift: regularity, duality and uniqueness, Electron. J. Probab., Vol. 24 (2019), No. 136, 1-72.

[2] M. G. Crandall, K. Fok, M. Kocan, and A. Święch, Remarks on nonlinear uniformly parabolic equations, Indiana Univ. Math. J., Vol. 47 (1998), No. 4, 1293-1326.

[3] E. B. Dynkin, "Markov processes", Fizmatgiz, Moscow, 1963 in Russian; English translation in Grundlehren Math. Wiss., Vols. 121, 122, Springer-Verlag, Berlin, 1965.

[4] L. Escauriaza, $W^{2, n}$ a priori estimates for solutions to fully non-linear equations, Indiana Univ. Math. J. 42 (1993), 413-423.

[5] E.B. Fabes and D.W. Stroock, The $L^{p}$-integrability of Green's functions and fundamental solutions for elliptic and parabolic equations, Duke Math. J., Vol. 51 (1984), No. 4, 997-1016.

[6] M.Giaquinta and M. Struwe, On the partial regularity of weak solutions of nonlinear parabolic systems, Mathematische Zeitschrift, Vol. 179 (1982), 437-451.

[7] D. Kinzebulatov and Yu. A. Semenov, Stochastic differential equations with singular (form-bounded) drift, arXiv:1904.01268.

[8] N.V. Krylov, On time inhomogeneous stochastic Itô equations with drift in $L_{d+1}$, http://arxiv.org/abs/2005.08831

[9] N.V. Krylov, "Controlled diffusion processes", Nauka, Moscow, 1977 in Russian; English transl. Springer, 1980.

[10] N.V. Krylov, On stochastic Itô processes with drift in $L_{d}$, http://arxiv.org/abs/2001.03660

[11] N.V. Krylov, On stochastic equations with drift in $L_{d}$, http://arxiv.org/abs/2001.04008

[12] N.V. Krylov and M.V. Safonov, An estimate of the probability that a diffusion process hits a set of positive measure, Doklady Academii Nauk SSSR, Vol.245 (1979), No.1, 18-20 in Russian, English transl. in Soviet Math. Dokl., Vol. 20 (1979), No. 2, 253-255.

[13] Kyeongsik Nam, Stochastic differential equations with critical drifts, arXiv:1802.00074 (2018).

[14] A.I. Nazarov, A centennial of the Zaremba-Hopf-Oleinik lemma, SIAM J. Math. Anal., Vol. 44 (2012), No. 1, 437-453.

[15] A.I. Nazarov, Interpolation of linear spaces and estimates for the maximum of a solution for parabolic equations, Partial differential equations, Akad. Nauk SSSR Sibirsk. Otdel., Inst. Mat., Novosibirsk, 1987, 50-72 in Russian; translated into English as On the maximum principle for parabolic equations with unbounded coefficients, https:// arxiv.org/abs/1507.05232

[16] Longjie Xie and Xicheng Zhang, Ergodicity of stochastic differential equations with jumps and singular coefficients, Annales de l'Institut Henri Poincaré - Probabilités et Statistiques, Vol. 56 (2020), No. 1, 175-229.

[17] Guohuan Zhao, Stochastic Lagrangian flows for SDEs with rough coefficients, arXiv:1911.05562v2. 
[18] Xicheng Zhang and Guohuan Zhao, Stochastic Lagrangian path for Leray solutions of 3D Navier-Stokes equations, arXiv:1904.04387.

Email address: nkrylov@umn.edu

127 Vincent Hall, University of Minnesota, Minneapolis, MN, 55455 